\title{
1 Conditional approach as cooperation in predator inspection: a role for serotonin?
}

2 Ana Flávia Nogueira Pimentel ${ }^{1,2}$, Tamires dos Santos Carvalho ${ }^{2}$, Fernando Lima ${ }^{2}$, Monica Lima-

3 Maximino $^{3}$, Marta Candeias Soares ${ }^{4}$, Caio Maximino ${ }^{1,2 *}$

4

$5{ }^{1}$ Laboratório de Neurociências e Comportamento "Frederico Guilherme Graeff", Universidade

6 Federal do Sul e Sudeste do Pará, Marabá/PA, Brazil

$7 \quad 2$ Faculdade de Psicologia, Universidade Federal do Sul e Sudeste do Pará, Marabá/PA, Brazil

$8{ }^{3}$ Laboratório de Neurofarmacologia e Biofísica, Universidade do Estado do Pará, Marabá/PA,

9 Brazil

$10{ }^{4}$ CIBIO, Centro de Investigação em Biodiversidade e Recursos Genéticos, Universidade do

11 Porto, Vairão, Portugal

$12 *$ Corresponding author

13 Caio Maximino

14 Laboratório de Neurociências e Comportamento - Instituto de Estudos em Saúde e Biológicas,

15 Universidade Federal do Sul e Sudeste do Pará, Unidade III

16 Av. dos Ipês, S/N, s/ CEP, Bairro Cidade Jardim, Marabá/PA, Brazil

17 cmaximino@unifesspa.edu.br 
Abstract

In guppies (Poecilia reticulata), a small number of individuals break away from a shoal and approach a potential predator, a behavior termed "predator inspection". These animals often

21 employ a "conditional approach" strategy, in which an individual approaches the predator in the

22 first move and subsequently approaches it only if a second individual swims even with it during

23 inspection. This strategy is analogous to the "tit-for-tat" strategy of the Prisoner's Dilemma,

24 suggesting that it could be used to study cooperation. Serotonin is thought to mediate cooperative

25 behavior in other fish species. Exposure to the animated image of a predator in a tank that

26 contained a parallel mirror - mimicking an equally cooperating conspecific - promoted

27 inspection and decreased refuge use, but increased freezing, suggesting that conditional approach

28 is also associated with fear. To understand whether serotonin participates in conditional approach

29 in guppies, we treated animals with either vehicle (Cortland's salt solution), fluoxetine (2.5

$30 \mathrm{mg} / \mathrm{kg}$ ) or metergoline $(1 \mathrm{mg} / \mathrm{kg})$, and tested then in a predator inspection paradigm. Fluoxetine

31 increased the time the animal spent inspecting the predator image, while metergoline decreased

32 it. Fluoxetine also decreased time spent avoiding the predator and increased freezing, while

33 metergoline decreased freezing. These results suggest that phasic increases in serotonin levels

34 promote conditional approach, suggesting a role for this neurotransmitter in cooperation.

35 Preprint: https://doi.org/10.1101/436345; Data and scripts: https://github.com/lanec-

36 unifesspa/TFT

37 Keywords: Serotonin; Cooperation; Conditional approach; Predator inspection; Guppy 


\section{Introduction}

39 Sometimes being seen is better than blending into the crowd. And when it comes to interactions

40 between putative preys and their predators, it may not be enough to stay vigilant as to simply

41 detect a predator more rapidly, but one also needs to signal its presence and awareness (e.g. head

42 bobbing and fin flicking [1]) and sometimes even to provide information regarding own physical

43 condition (e.g. leaping in artiodactyls [2]). Indeed, predators seem to be less successful when

44 prey are fully aware of their presence, in part due to the improved vigilance effect in which

45 groups are able to detect predators sooner than solitary individuals [3]. One seemingly

46 paradoxical behaviour occurs when some of these prey leave the shoal to slowly approach and

47 inspect the predator presumably to reduce the uncertainty of the situation and to be able to gain

48 valuable information to all other conspecifics involved [4]. Since this behaviour implies costs to

49 the individual(s) that interacts with the predator but contributes to the fitness of the shoal, it has

50 been interpreted as cooperative, however, not without strategic specificities.

Conditional approach is a behavioural strategy used by different fish species, including guppies Poecilia reticulata [5-8] $\square$ and sticklebacks Gasterosteus aculeatus $[9,10]$, and acts as an incentive for predator inspection, given that the risk of approaching a predator is shared between all inspectors, but not with the individuals that keep a distance $[11,12]$. Conditional approach represents a cooperative strategy that is comparable to a Prisoner's Dilemma game $[13,14]$, in which cheating a partner (by staying behind) is the most profitable option while joint inspection is the most beneficial (symmetrical) form of cooperation, allowing the advantages of inspecting the predator to overcome those of remaining at a safe distance [11]. Trivers [15]

59 proposed the Prisoner's Dilemma as a mechanism to interpret the evolution of reciprocal 60 altruism, and Axelrod and Hamilton [14] showed that "tit-for-tat" is an evolutionary stable 61 strategy. This strategy instructs the player to cooperate in the first move and to copy subsequent 
62 moves by the partner. Tit-for-tat requires a pre-specified payoff matrix that is, in the case of

63 conditional approach, unknown [5]; however, conditional approach is analogous to tit-for-tat in

64 that when fish inspect in pairs, due to the higher risk of taking the lead, exchanges in leading

65 position are an example of cooperation based on reciprocity [5,6]. Prisoner's Dilemma therefore

66 models a situation in which each individual receives a worse pay-off for not cooperating than for

67 cooperating $[12,14,15]$. In conditional approach, non-cooperating individuals also obtain

68 information regarding the predator without incurring in risk, but mutual reciprocal cooperation

69 offers animals a better pay-off. Conditional approach is only employed by inspecting individuals

70 in cooperative partnerships; predator inspection per se can be made by solitary individuals, as

71 has been shown for guppies [16,17] and zebrafish (Danio rerio) $[18,19]$. Predator inspection by

72 singletons is still cooperative, as the rest of the shoal can benefit from the transmission of

73 information about the predator, but involves inspection only by one individual $[17,20]$.

74 The guppy is a small ovoviviparous fish originating from Central America and northern

75 South America [21]. It is well known by its bright colours, and widely used in ornamental

76 aquaria. Seghers [22] observed that some guppies, when facing a predator at a distance in the

77 wild, soon inspect this potential threat. Guppies leave the shoal individually or in small groups,

78 and approach the potential threat to obtain information. There is evidence that guppies use

79 conditional approach during this inspection behaviour [5-7,20,23,24]. Dugatkin [5] suggested

80 that conditional approach presents the basic characteristics of tit-for-tat: it is "nice" (that is, the

81 first "move" is to cooperate by initiating inspection), "retaliatory" (animals defect by retreating

82 from inspection if the conspecific does not reciprocate), and "forgiving" (animals cooperate by

83 re-initiating inspection if a previously defecting conspecific changes its course and begins

84 inspection).

85 Serotonin (5-hydroxytryptamine, 5-HT) is a monoamine that is synthesized from

86 tryptophan. This monoamine participates in the modulation of stress and defensive behaviour in 
87 fish [25-27], and has been implicated in vertebrate social behaviour [27,28]. Converging

88 evidence from human and non-human animal research suggest that altering 5-HT levels can

89 directly influence social perception and mood, with decreased serotonin leading to isolation and

90 decreased sociality [29]. Moreover, it has been shown that 5-HT participates directly in pro-

91 social and cooperative behaviour: tryptophan depletion reduces cooperation in the Prisoner's

92 Dilemma in human participants, and decreases the probability of cooperative responses given

93 previous mutually cooperative behaviour [30]. In the cleaner wrasse Labroides dimidiatus, acute

94 treatment with fluoxetine or the 5-HT $1 \mathrm{~A}$ receptor agonist ( \pm )-8-Hydroxy-2-

95 (dipropylamino)tetralin (8-OH-DPAT) increased client inspection and tactile stimulation, while

96 treatment with the tryptophan hydroxylase inhibitor para-chlorophenylalanine or the 5-HT

97 receptor

antagonist

N-[2-[4-(2-Methoxyphenyl)-1-piperazinyl]ethyl]-N-2-

98 pyridinylcyclohexanecarboxamide (WAY 100,635) decreased client inspection and cleaners'

99 cheating levels [31]. WAY 100,635 also delays learning of client value, suggesting that this

100 receptor is involved in the perception of danger and therefore in how cleaners appraise, acquire

101 information and respond to client-derived stimuli [32].

102 Given that predator inspection entails the evaluation of the level of predator threat, as

103 well as of the value of cooperating vs. defecting with conspecifics, it is probable that 5-HT is

104 involved in the mediation of conditional approach/cooperative behaviour in guppies. We

105 modified the method described by Dugatkin [5], using a video animation of a sympatric predator

106 (the blue acará cichlid Aequidens pulcher) presented to a single guppy in a tank with a parallel

107 mirror. We predicted that the predator animation, compounded with the parallel mirror -

108 mimicking an equally cooperating conspecific, with approaches and retreats to the predator -,

109 would elicit predator approach (inspection), leading animals to spend more time in the inspection

110 section than near the predator rather than in the avoidance zone or in the refuge. We also 
111 predicted that the predator animation would elicit fear-like behaviour, including freezing, as well

112 as higher time spent in the avoidance zone and in the refuge. The first prediction was confirmed,

113 but the second prediction was only partially true, given that the animated image increased

114 freezing, but not time in the avoidance zone, and decrased time in the refuge zone. We also tested

115 the hypothesis that serotonin promotes conditional approach by treating animals with fluoxetine

116 or metergoline, a non-selective serotonin receptor antagonist. We predicted that phasic 5-HT

117 (increased by acute fluoxetine) would promote conditional approach, while simultaneously

118 increasing fear/anxiety (as observed in zebrafish: [33]); blocking receptors with metergoline

119 (thereby decreasing the role of tonic 5-HT) would decrease conditional approach and decrease

120 fear. The first prediction was confirmed, but metergoline only decreased freezing, without

121 affecting conditional approach.

122

\section{2. Methods}

124 2.1. Animals, housing, and baseline characteristics

125 For the experiments of the present research, 46 male guppies were used. Animals were

126 bought from a commercial vendor, and left to acclimatize to the laboratory (LaNeC) for two

127 weeks before beginning experiments. The animals were bred at the vendor, and represent the

128 third generation from populations captured in the wild in the Amazon (Belém/PA, Brazil). These

129 domesticated animals represent a mix of strains. Animals were fed daily with commercial feed.

130 The animals were housed collectively in $40 \mathrm{~L}$ tanks, at a maximum of 25 fish per tank, with the

131 following water quality parameters being controlled: dissolved oxygen $\approx 7.8 \mathrm{mg} / \mathrm{L}$, ammonia

$132<0.002 \mathrm{mg} / \mathrm{L}, \mathrm{pH} 7.0-8.0$, temperature $24-30{ }^{\circ} \mathrm{C}$.

133

134 2.2. Induction of conditional approach 
136 extreme ("refuge zone") and a computer screen (Samsung T20c310lb, 20”, LED screen, nominal

137 brightness $20 \mathrm{~cd} / \mathrm{m}^{3}$ ) positioned on the opposite extreme. A mirror was positioned in parallel to

138 one of the sides of the tank for the whole duration of experiments. The avoidance zone is defined

139 as the half of the tank that is farthest from the screen, minus the refuge zone, and the inspection

140 zone is defined as the other half of the tank (Figure 1). The avoidance zone, therefore, did not

141 include the refuge. While the mirrored image does not respond in the way a live individual would

142 (i.e. there is no exchange of the leading position, which is the position entailing more risk), and

143 therefore the mirrored image can only be as cooperative as the focal individual, using a mirror to

144 simulate cooperation has been shown to work and in fact reflect the cooperativeness of a specific

145 individual, despite the limitations in mimicking a natural behavior $[5,9]$.

146 Conditional approach was induced by playback of an animated predator (blue acará

147 cichlid Aequidens pulcher), produced and presented using LibreOffice Impress (Version: 5.3.7.2)

148 on a CPU running Linux 4.15 (Xubuntu 16.04). The animation was presented at the computer

149 screen for the last 6 min of each session, with the exception of the "Predator absent" condition of

150 Experiment 1 . The animals are expected to respond to acará cichlids with antipredator behavior,

151 which has been observed in other populations (e.g. ref. [34]).

152

153 2.3. Open science practices

154 The experiments were not formally pre-registered. Datasets and scripts are available from

155 https://github.com/lanec-unifesspa/TFT (doi: 10.5281/zenodo.1290044).

157 2.4. Experiment 1

$158 \quad$ 2.4.1. Experimental design, endpoints, and statistical analysis 
160 groups: "Predator absent" and "Predator present". For both groups, the mirror was positioned in

161 parallel to the side of the tank. In the first group, animals were introduced to the tank and th

162 allowed to explore it for $10 \mathrm{~min}$; in the 5 minute, the video was not turned on. The second

163 group was subjected to the same manipulations, except that the video was turned on in the 5

164 minute. The order of subjects was randomized using http://randomization.com/. Video recordings

165 of the behaviour were made and later analyzed with manual event recording, with the help of the

166 software X-Plo-Rat 2005 (https://github.com/lanec-unifesspa/x-plo-rat [35]) by two trained

167 observers. Observers were not blind to group allocation, as it was not possible to exclude the

168 portion of the video that included the monitor. Times spent in the avoidance, refuge, or

169 inspection zone were computed as percent change, as follows:

$$
\frac{\text { Timespentatzoneforthelast } 5 \mathrm{~min}-\text { Timespentatzoneforthefirst } 5 \mathrm{~min}}{\text { Timespentatzoneforthefirst } 5 \mathrm{~min}} \times 100 \%
$$

173 values $<0.05$ considered statistically significant. Effect sizes for these variables were reported as

174 Cohen's $d$. Raw values for the acclimation period were also analyzed, to understand whether

175 there were biases or baseline differences across groups that could be responsible for possible 176 effects.

177 Data on freezing was analyzed as raw values, in $s$, of the time spent freezing. These data 178 were analyzed using a repeated measures ANOVA, with time block as within-subjects effect, and 179 group as between-subjects effect. Effect sizes for freezing were reported as $\omega$. 


\subsection{Experiment 2}

\subsubsection{Drug treatments}

Fluoxetine hydrochloride (CAS \#54910-89-3) was bought from Libbs. Metergoline (CAS

$184 \# 17692-51-2)$ was bought from Virbac Brasil. All treatments were acute - i.e. a single injection

185 was made, 20 min. before experiments. The fluoxetine dose was based on previous work with

186 zebrafish [36], which showed an antipanic-like effect of this dose in an acute treatment. The

187 metergoline dose was based on previous work with rats [37], which showed an effect of this dose

188 on the forced swimming test.

189 Injection procedure was adapted from a protocol existing for intraperitoneal

190 administration in zebrafish [38]. Briefly, animals were anesthetized in cold water $\left(12{ }^{\circ} \mathrm{C}-17^{\circ} \mathrm{C}\right)$,

191 and transferred to a "surgical bed" made of a washing sponge with a through that allowed

192 positioning the animal with the ventral side exposed, while simultaneously perfusing the gills.

193 The sponge was soaked with ice-cold water to maintain gill perfusion, and the setup was kept on

194 ice. Drugs were injected at a volume of $2 \mu \mathrm{L} /$ animal, using a Hamilton microsyringe (701N,

195 needle gauge 26s), and animals were immediately transferred to aerated water at room

196 temperature to recover.

$198 \quad$ 2.5.2. Experimental design and statistical analysis

199 In the second experiment, 30 animals were used, divided equally and randomly between 200 three groups: a control group (CTRL), injected with vehicle (Cortland's salt solution; 201 https://wiki.zfin.org/display/prot/Cortland\%27s+salt+solution); a fluoxetine-treated group

202 (FLX), injected with $2.5 \mathrm{mg} / \mathrm{kg}$ fluoxetine; and a metergoline-treated group (MET), injected with $2031 \mathrm{mg} / \mathrm{kg}$ metergoline. The dose was based on the average weight of the animals. Experimenters

204 were blinded to treatment by using coded vials for drugs. 20 min after injection, animals were 205 individually transferred to the test tank, and subjected to the same manipulations as the EXP 
206 group from Experiment 1. The order of subjects was randomized using

207 http://randomization.com/. Data were recorded and registered as described in Experiment 1, with

208 spatiotemporal endpoints (time spent in avoidance, refuge, and inspection zones) indexed as

209 percent change, and data for freezing analyzed raw. Spatiotemporal data was analyzed using

210 Kruskal-Wallis' rank sum test followed by Dunn's post-hoc multiple comparison tests adjusted

211 with the Benjamini-Hochberg method; freezing data was analyzed using a repeated measures

212 ANOVA, with time block as within-subjects effect, and treatment as between-subjects effect.

213 Effect sizes for freezing were reported as $\omega^{2}$.

\section{3. Results}

\section{3.1. Experiment 1}

No differences were found in baseline (pre-animation) values across groups on time in

218 the refuge $(\mathrm{W}=23, \mathrm{p}=0.3706$; data not shown), avoidance zone $(\mathrm{W}=29, \mathrm{p}=0.7984$; data not

219 shown), or inspection zone ( $\mathrm{W}=40, \mathrm{p}=0.4418$; data not shown). The animated predator

220 increased time in the inspection zone when the mirror was parallel to the tank (Figure $2 \mathrm{~A} ; \mathrm{W}=$

221 13, $\mathrm{p}=0.0498, d=-1.25)$. The animated predator did not change time spent in the avoidance

222 zone (Figure $2 \mathrm{~B} ; \mathrm{W}=24, \mathrm{p}=0.4418, d=-0.49$ ). Refuge use was altered by the animated

223 predator (Figure $2 \mathrm{C}$; $\mathrm{W}=59, \mathrm{p}=0.003, d=0.82$ ). Finally, the animated predator increased

224 freezing in the second time block (i.e. after the stimulus was turned on), but not in the first time

block (Figure 2D; $\mathrm{F}_{[1,28]}=6.46, \mathrm{p}=0.0169, \omega^{2}=0.138$ for the main effect of group).

\subsection{Experiment 2}

Acute fluoxetine increased time spent in the inspection zone $(Z=-2.16, p=0.046$ vs. 229 control), while metergoline decreased time in the inspection zone $(Z=2.01, p=0.045$ vs. 
230 control $)\left(\right.$ Figure $\left.3 \mathrm{~A} ; \chi_{[\mathrm{df}=2]}^{2}=17.36, \mathrm{p}=0.00017, \omega^{2}=0.476\right)$. Only fluoxetine produced an

231 effect on time spent in the avoidance zone (Figure $3 \mathrm{~B} ; \chi_{[\mathrm{df}=2]}^{2}=16.059, \mathrm{p}=0.00033, \omega^{2}=-$

232 0.555), with fluoxetine decreasing time spent in the avoidance zone $(Z=2.616, p=0.013$ vs.

233 control), and metergoline having no effect $(\mathrm{Z}=-1.32, \mathrm{p}=0.186$ vs. control). Neither fluoxetine

234 nor metergoline altered refuge use (Figure $3 \mathrm{C} ; \chi_{[\mathrm{df}=2]}^{2}=1.319, \mathrm{p}=0.517, \omega^{2}=-0.00331$ ). $-7$

235 Freezing was increased by fluoxetine $(\mathrm{p}=4.0 \cdot 10$ vs. control), while metergoline decreased it ( $p$ $-7$

$236<4.0 \cdot 10$ vs. control)(Figure 3D; $\mathrm{F}_{[2,27]}=26, \mathrm{p}=5.2 \cdot 10, \omega=0.255$ for the interaction

237 term).

4. Discussion

In the present work, we replicated the observation made by Dugatkin [5,6]on conditional approach by guppies during predator inspection, and extended his findings by showing that guppies also freeze more during predator inspection. We also show that phasic serotonin promotes conditional approach/cooperation but increases freezing/fear, and limited evidence was found for a tonic participation of serotonin in conditional approach.

We predicted that the animated predator image would promote predator inspection, but would also increase fear, reflected by increased freezing, as well as time spent in the avoidance 247 and refuge zones. While freezing and refuge use were indeed increased, animals did not appear 248 to avoid the predator. We suggest that freezing, in this situation, is an optimal strategy to avoid 249 being detected by the predator while at the same time benefiting from gathering information, 250 maintaining a high level of alertness. The relationship between fear, stress, and social behaviour 
251 has seldomly been addressed in the literature [27,39]. There is some evidence from cleaning

252 gobies Elacatinus spp. that cooperating in cleaning mutualisms with piscivorous clients

253 (potential predators) is stressful, as cortisol levels are increased during these interactions [40].

254 Interestingly, field experiments in cleaner wrasse showed that treament with cortisol increases

255 "cheating", in which cleaners provide small clients with more tactile stimulation with their

256 pectoral and pelvic fins - a behaviour that attracts larger clients that are then bitten to obtain

257 mucus -, while blocking glucocorticoid receptors led cleaners to increase tactile stimulation to

258 larger clients [41].

An important concern of our experiments is that, differently from Dugatkin [5], the

260 animals used in Experiments 1 and 2 are not wild-caught, but laboratory-reared, and the stimulus

261 predator was the blue acará cichlid. As a result, animals could have been displaying behavior that

262 is not specifically anti-predator in nature, but instead represent a "curiosity" approach to any

263 novel fish stimulus. Indeed, in wild populations from Trinidad, pike cichlids (Crenicichla

264 lepidota) induce a "surfacing" behavior that is not observed when animals are exposed to acará

265 cichlids [34]; however, even in wild populations, acará and pike cichlids do not differ in their

266 ability to induce an inspection response or in their ability to induce fear-like behavior (e.g. inhibit

267 foraging and increase shoaling) [34]. While wild-caught female guppies from high-predation

268 areas appear to respond less to blue acará cichlids than to pike cichlids $C$. frenata, this difference

269 disappears at the first and second generations derived from these wild populations [42]. In the

270 light of these findings, and considering the effect that our blue acará cichlid animation had on

271 freezing, our results suggest that this domesticated population is displaying normal antipredator

272 behavior, and not simply a novelty response. However, a neophobic response cannot be fully

273 discarded as driving part of the behavior reported here.

An alternative explanation to the results from Experiment 1 is that predator inspection

275 does not represent conditional approach, but instead males undertake inspection as a 
276 demonstration to females of their superiority in relation to non-inspectors. Indeed, it has been

277 demonstrated that female guppies prefer bolder males, which show higher levels of inspection

278 [43,44], and bolder individuals tend to produce more social interactions [45]; assortative

279 interactions and sexual selection are expected to play a role in the establishment of this strategy

280 [8]. However, this is not necessarily contradictory with the conditional approach strategy since,

281 differently from "pure" tit-for-tat conditional approach does not require some form of kin or

282 group selection to be at work. Conditional approach can be better understood in a "social

283 competence" approach [12,46], in which situation-specific cues (such as presence or absence of

284 females, or varying risk levels), together with various other factors, allow individuals to assess

285 the situation in order to make behavioral decisions. While risk taking/boldness certainly takes

286 part on this [4], it is not the only variable responsible for the development of predator inspection

287 in our paradigm.

In Experiment 2, we hypothesized tonic and phasic roles for serotonin in promoting

cooperation and increasing fear. We found that acute fluoxetine increased inspection and fear,

290 while acute metergoline decreased both. Guppies under treatment with fluoxetine spent more

291 time in the inspection zone (i.e. nearer to the image of a predator) and less in the avoidance zone.

292 However, fluoxetine also increased freezing, suggesting that its effects were not fully explained

293 by simply increasing "boldness" (or, conversely, decreasing fear and/or anxiety). In the cleaner

294 wrasse, fluoxetine increased the probability of cleaning clients without altering cleaning quality

295 [31], suggesting that phasic serotonin increases the motivation to cooperate in fish [47].

296 Differently from the cleaner wrasse, however, conditional approach during predator inspection

297 entails exposure to threat, while most of the clientele of cleaners is harmless. Acute fluoxetine

298 increased freezing, while metergoline decreased it, suggesting that serotonin controls 
antipredator behaviour in guppies. Similar observations have been made in zebrafish, in which

300 serotonin appears to have a "dual role" in mediating responses to potential vs. real threat [25].

301

While phasic serotonin appears to motivate cooperation in guppies, we found evidence of

302 a serotonergic tone promoting conditional approach. This is consistent with other findings from

303 the literature: tryptophan depletion reduces cooperation in the Prisoner's Dilemma in human

304 participants [30], and 5-HT receptor antagonism or eliminating 5-HT tonus decreases cleaning $1 \mathrm{~A}$

305 in cleaner wrasses [31]. In this last case, reducing 5-HT activity also decreased cheating

306 frequencies due to an overall reduction of the proportion of clients inspected and of the average

307 duration of interactions [31]. These effects may occur via mediation of risk perception, which

308 would enhance the cleaner's appraisal of the threat represented by predatory clients $309[27,39,47,48]$.

\section{5. Conclusion}

Overall, the results reported present a scenario in which serotonin acts to promote

313 cooperation in a laboratory setting in fish. These results contribute to the description of the

314 neurochemical bases of pro-social behaviour, and could have translational applications.

315 Moreover, they also shed light on the relationship between fear and sociality, an area seldomly

316 explored in the social behaviour literature $[27,39]$. Whether this is also modulated by mediators

317 of fear and stress, such as cortisol, is still unknown. 
AFNP is the recipient of a CNPq/PIBITI scholarship. $\mathrm{CM}$ is the recipient of a

$321 \mathrm{CNPq} /$ Universal grant (\#400726/2016-5). MCS is the recipient of two FCT grants

322 (SFRH/BPD/109433/2015; PTDC/MAR/105276/2008).

[1] G.E. Brown, J.G. Godin, J. Pedersen, Fin-flicking behaviour: A visual antipredator alarm signal in a characin fish, Hemigrammus erythrozonus, Anim. Behav. 58 (1999) 469-475. doi:10.1006/anbe.1999.1173.

[2] T. Caro, W.L. Allen, Interspecific visual signalling in animals and plants: A functional classification, Philos. Trans. R. Soc. B Biol. Sci. 372 (2017) 20160344. doi:10.1098/rstb.2016.0344.

[3] T. Caro, Antipredator defenses in birds and mammals, University of Chicago Press,

333 [4] L.A. Dugatkin, The evolution of risk-taking, Cerebrum. (2013).

L.A. Dugatkin, Do guppies play TIT FOR TAT during predator inspection visits?, Behav. Ecol. Sociobiol. 23 (1988) 395-399. doi:10.1007/BF00303714.

L.A. Dugatkin, Dynamics of the TIT FOR TAT strategy during predator inspection in the guppy (Poecilia reticulata), Behav. Ecol. Sociobiol. 29 (1991) 127-132. doi:10.1007/BF00166487.

L.A. Dugatkin, Tendency to inspect predators predict mortality risk in the guppy, Poecilia reticulata, Behav. Ecol. 3 (1992) 124-128.

[8] M. Edenbrow, B.H. Bleakley, C.R. Tyler, I.W. Ramnarine, D.P. Croft, The evolution of cooperation: Interacting phenotypes among social partners, Am. Nat. 189 (2017) 630-643. doi:10.1086/691386.

[9] M. Milinski, TIT FOR TAT in sticklebacks and the evolution of cooperation, Nature. 325 (1987) 433-435.

[10] F.A. Huntingford, J. Lazarus, B.D. Barrie, S. Webb, A dynamic analysis of cooperative predator inspection in sticklebacks, Anim. Behav. 47 (1994) 413-423.

[11] L.A. Dugatkin, The evolution of cooperation, Bioscience. 47 (1997) 355-362. framework of social competence, Curr. Opin. Behav. Sci. 3 (2015) 31-37. doi:10.1016/j.cobeha.2015.01.008. 
352 [13] R.D. Luce, H. Raiffa, Games and decision: Introduction and critical survey, Wiley, New York, NY, 1957.

[14] R. Axelrod, W. Hamilton, The evolution of cooperation, Science (80-. ). 211 (1981) 13901396. doi:10.1126/science.7466396.

[15] R.L. Trivers, The evolution of reciprocal altruism, Q. Rev. Biol. 46 (1971) 35-57. doi:10.1086/406755.

[16] A.E. Magurran, B.H. Seghers, Population differences in predator recognition and attack cone avoidance in the guppy Poecilia reticulata, Anim. Behav. 40 (1990) 443-452.

[17] L.A. Dugatkin, J.-G.J. Godin, Predator inspection, shoaling and foraging under predation hazard in the Trinidadian guppy, Poecilia reticulata, Environ. Biol. Fishes. 34 (1992) 265276. doi:10.1007/BF00004773.

363

[18] L.A. Dugatkin, M.A. McCall, R.G. Gregg, A. Cavanaugh, C. Christensen, M. Unseld, B. Sciences, V. Sciences, Zebrafish (Danio rerio) exhibit individual differences in risk-taking behavior during predator inspection, Ethology, Ecol. Evol. 17 (2010) 77-81.

366

367

[19] M. Dadda, A. Domenichini, L. Piffer, F. Argenton, A. Bisazza, Early differences in 368 epithalamic left-right asymmetry influence lateralization and personality of adult zebrafish, Behav. Brain Res. 206 (2010) 208-215. doi:10.1016/j.bbr.2009.09.019.

371

[20] L.A. Dugatkin, J. Godin, Prey approaching predators: A cost-benefit perspective, Ann. Zool. Fenn. 29 (1992) 233-252.

372

[21] A.E. Magurran, Evolutionary ecology - The Trinidadian guppy, Oxford University Press, Oxford, UK, 2005.

374

B.H. Seghers, Analysis of geographic variation in the antipredator adaptations of the guppy, Poecilia reticulata, University of British Columbia, 1973. doi:10.14288/1.0100947.

[23] L.A. Dugatkin, M. Alfieri, Guppies and the TIT FOR TAT strategy: Preference based on past interaction, Behav. Ecol. Sociobiol. 28 (1991) 243-246. doi:10.1007/BF00175096.

[24] L.A. Dugatkin, M.S. Alfieri, Interpopulational differences in the use of the Tit- For-Tat strategy during predator inspection in the guppy, Poecilia reticulata, Evol. Ecol. 6 (1992) 519-526. doi:10.1007/BF02270695.

381

[25] A.M. Herculano, C. Maximino, Serotonergic modulation of zebrafish behavior: Towards a paradox, Prog. Neuropsychopharmacol. Biol. Psychiatry. 55 (2014) 50-66. doi:10.1016/j.pnpbp.2014.03.008. Zool. 62 (2016) 317-323. doi:10.1093/cz/zow037. 
386 [27] M.C. Soares, R. Gerlai, C. Maximino, The integration of sociality, monoamines and stress neuroendocrinology in fish models: Applications in the neurosciences, J. Fish Biol. 93 (2018) 170-191. doi:10.1111/jfb.13757.

[28] T.R. Insel, J.T. Winslow, Serotonin and neuropeptides in affiliative behaviors, Biol. Psychiatry. 44 (1998) 207-219. doi:10.1016/S0006-3223(98)00094-8.

[29] D. Kiser, B. SteemerS, I. Branchi, J.R. Homberg, The reciprocal interaction between serotonin and social behaviour, Neurosci. Biobehav. Rev. 36 (2012) 786-798. doi:10.1016/j.neubiorev.2011.12.009.

[30] R.M. Wood, J.K. Rilling, A.G. Sanfey, Z. Bhagwagar, R.D. Rogers, Effects of tryptophan depletion on the performance of an Iterated Prisoner's Dilemma game in healthy adults, Neuropsychopharmacology. 31 (2006) 1075-1084. doi:10.1038/sj.npp.1300932.

[31] J.R. Paula, J.P. Messias, A.S. Grutter, R. Bshary, M.C. Soares, The role of serotonin in the modulation of cooperative behavior, Behav. Ecol. 26 (2015) 1005-1012. doi:10.1093/beheco/arv039.

400

[32] M.C. Soares, S.C. Cardoso, J.T. Malato, J.P.M. Messias, Can cleanerfish overcome 401 temptation? A selective role for dopamine influence on cooperative-based decision making, Physiol. Behav. 169 (2017) 124-129. doi:10.1016/j.physbeh.2016.11.028.

404

[33] C. Maximino, B. Puty, R. Benzecry, J. Araujo, M.G. Lima, E. de J.O. Batista, K.R.M. Oliveira, M.E. Crespo-López, A.M. Herculano, Role of serotonin in zebrafish (Danio rerio) anxiety: Relationship with serotonin levels and effect of buspirone, WAY 100635,

[34] M.S. Botham, C.J. Kerfoot, V. Louca, J. Krause, The effects of different predator species SB 224289, fluoxetine and para-chlorophenylalanine (pCPA) in two behavioral models, on antipredator behavior in the Trinidadian guppy, Poecilia reticulata, Naturwissenschaften. 93 (2006) 431-439. doi:10.1007/s00114-006-0131-0.

J. Tejada, K.T.C. Correio, S. Morato, X-Plo-Rat: Automatic scoring of behavior in delimited spaces, Psicol. Teor. e Pesqui. 33 (2018) e3322. doi:10.1590/0102.3772e3322. WAY 100,635 dissociate increases in scototaxis and analgesia induced by conspecific alarm substance in zebrafish (Danio rerio Hamilton 1822), Pharmacol. Biochem. Behav. 124C (2014) 425-433. doi:10.1016/j.pbb.2014.07.003. 
422 [39] M.C. Soares, S.C. Cardoso, T. dos S. Carvalho, C. Maximino, Using model fish to study the biological mechanisms of cooperative behaviour: A future for translational research concerning social anxiety disorders?, Prog. Neuro-Psychopharmacology Biol. Psychiatry.

[40] M.C. Soares, R.R.R. Bshary, S.C. Cardoso, I.M. Côté, R.F. Oliveira, Face your fears:

427 Cleaning gobies inspect predators despite being stressed by them, PLoS One. 7 (2012) e39781. doi:10.1371/journal.pone.0039781.

[41] M.C. Soares, S.C. Cardoso, A.S. Grutter, R.F. Oliveira, R. Bshary, Cortisol mediates cleaner wrasse switch from cooperation to cheating and tactical deception, Horm. Behav. 66 (2014) 346-350. doi:10.1016/j.yhbeh.2014.06.010.

[42] J.L. Kelley, A.E. Magurran, Effects of relaxed predation pressure on visual predator recognition in the guppy, Behav. Ecol. Sociobiol. 54 (2003) 225-232. doi:10.1007/s00265-003-0621-4.

[43] J.G. Godin, L.A. Dugatkin, Female mating preference for bold males in the guppy,

[44] D.P. Croft, R. James, P.O.R. Thomas, C. Hathaway, D. Mawdsley, K.N. Laland, J. Krause, Social structure and co-operative interactions in a wild population of guppies (Poecilia reticulata), Behav. Ecol. Sociobiol. 59 (2006) 644-650. doi:10.1007/s00265-005-0091-y.

[45] D.P. Croft, J. Krause, S.K. Darden, I.W. Rammarine, J.J. Faria, R. James, Behavioural trait assortment in a social network: Patterns and implications, Behav. Ecol. Sociobiol. 63 (2009) 1495-1503. doi:10.1007/s00265-009-0802-x.

[47] M.C. Soares, The neurobiology of mutualistic behavior: The cleanerfish swims into the 


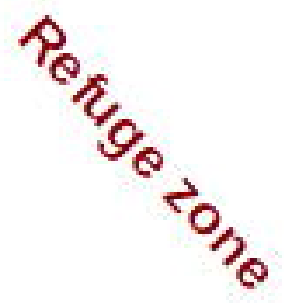

Avoidance zone

Inspection zone
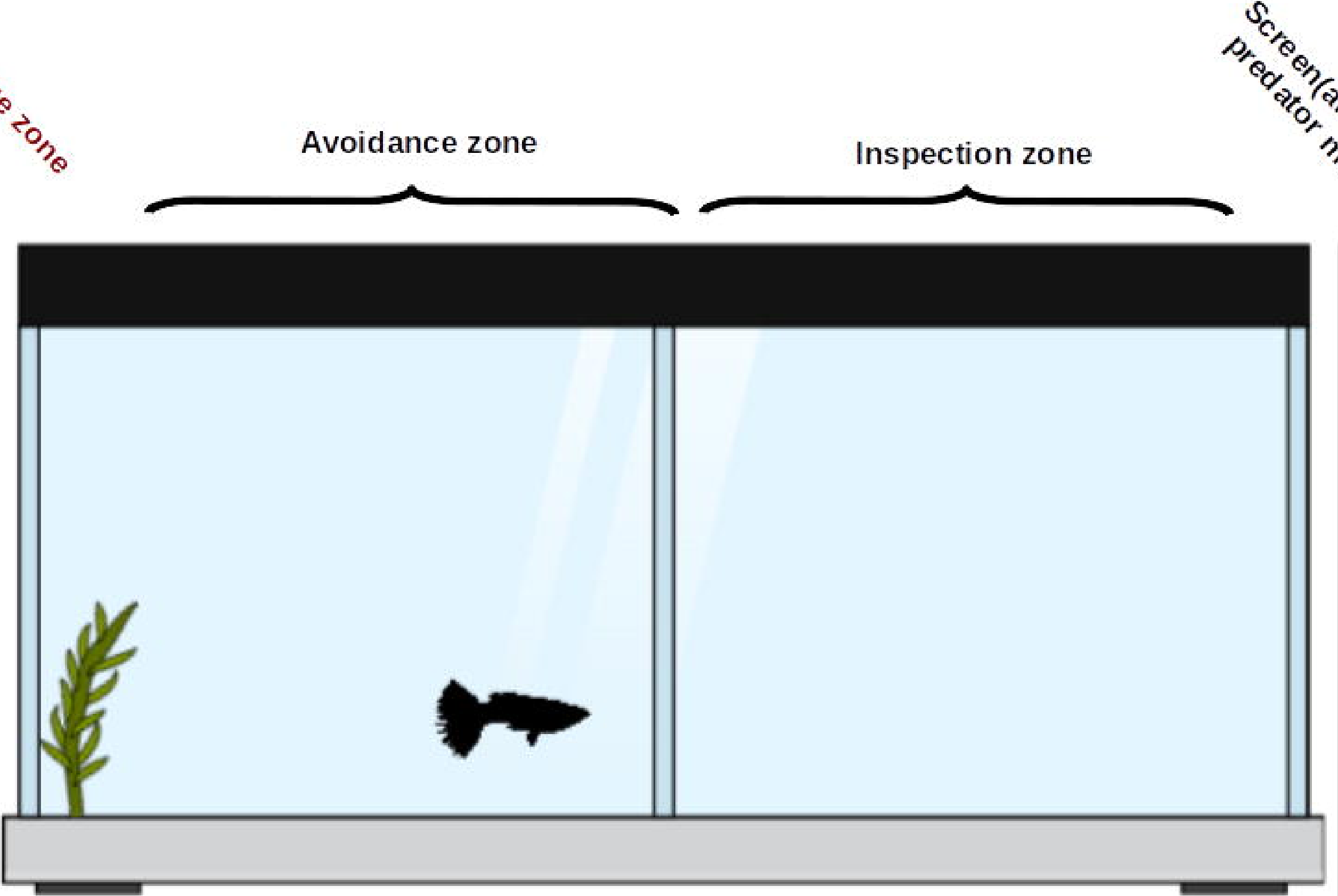


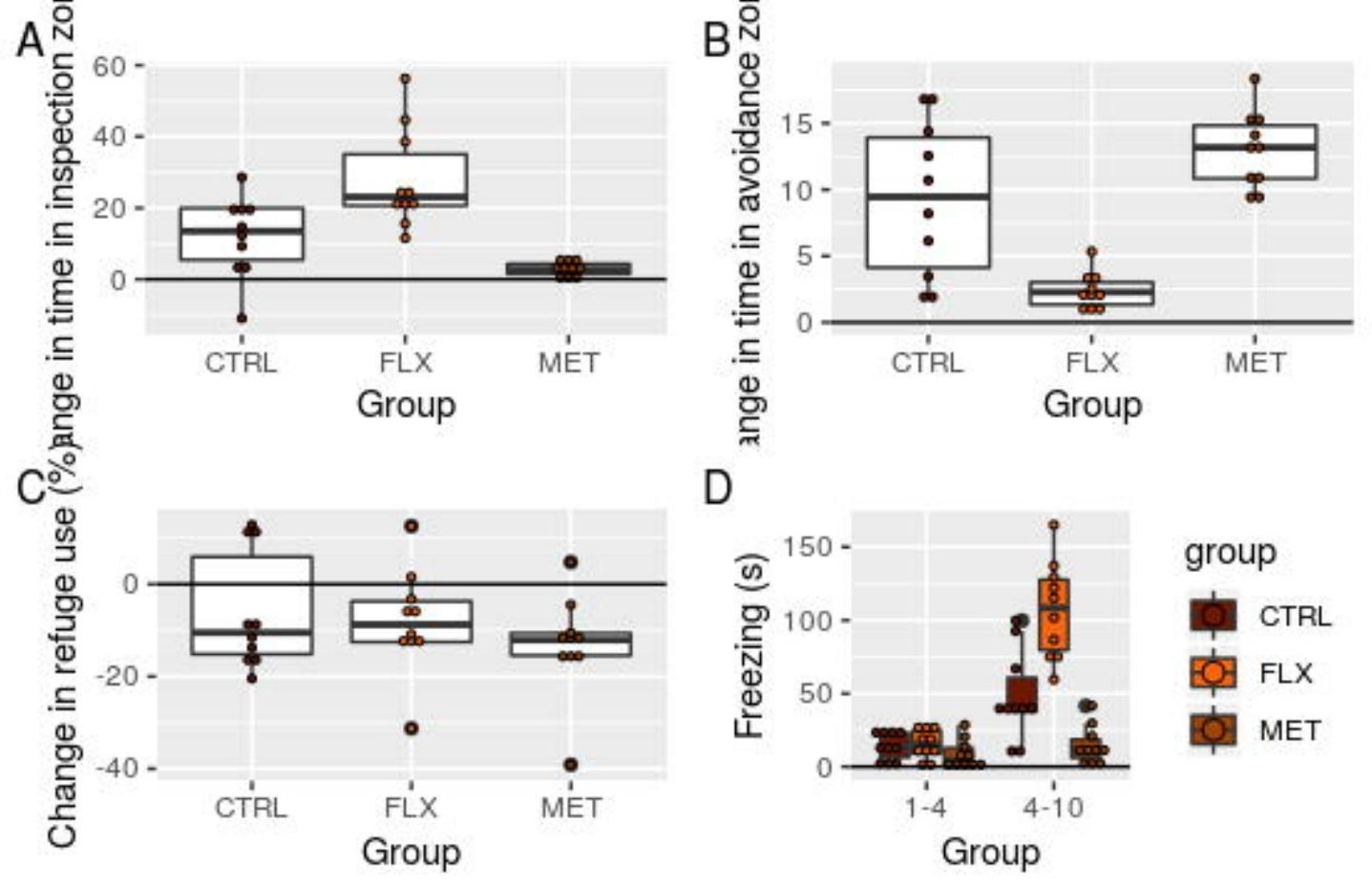

$A_{\sim}^{N}$
$B$

D group

CTRL 우 FLX 우 MET 